\title{
Quasi-Bézier Curves with Shape Parameters
}

\author{
Jun Chen \\ Faculty of Science, Ningbo University of Technology, Ningbo 315211, China \\ Correspondence should be addressed to Jun Chen; chenjun88455579@163.com
}

Received 2 October 2012; Revised 7 February 2013; Accepted 24 February 2013

Academic Editor: Juan Manuel Peña

Copyright (C) 2013 Jun Chen. This is an open access article distributed under the Creative Commons Attribution License, which permits unrestricted use, distribution, and reproduction in any medium, provided the original work is properly cited.

\begin{abstract}
The universal form of univariate Quasi-Bézier basis functions with multiple shape parameters and a series of corresponding QuasiBézier curves were constructed step-by-step in this paper, using the method of undetermined coefficients. The series of Quasi-Bézier curves had geometric and affine invariability, convex hull property, symmetry, interpolation at the endpoints and tangent edges at the endpoints, and shape adjustability while maintaining the control points. Various existing Quasi-Bézier curves became special cases in the series. The obvious geometric significance of shape parameters made the adjustment of the geometrical shape easier for the designer. The numerical examples indicated that the algorithm was valid and can easily be applied.
\end{abstract}

\section{Introduction}

The Bézier curve $\gamma_{1}(t)$ listed as follows has a direct-viewing structure and can be computed using a simple process; it is also one of the most important tools in computer-aided geometric design (CAGD). Consider

$$
\gamma_{1}(t)=\sum_{i=0}^{n} \mathbf{P}_{i} B_{i}^{n}(t), \quad t \in[0,1]
$$

Here, Bernstein basis functions $\left\{B_{i}^{n}(t)\right\}_{i=0}^{n}$ are defined as:

$$
B_{i}^{n}(t)=\left(\begin{array}{c}
n \\
i
\end{array}\right)(1-t)^{n-i} t^{i}, \quad i=0,1, \ldots, n .
$$

Given that the shape of the curve is characterized by the control polygon, the designer always adjusts the control point $\left\{\mathbf{P}_{i}\right\}_{i=0}^{n}$ when necessary. However, in the actual process, designing the geometrical shape is usually not completed at one time. The designer prefers to have more satisfactory geometrical shapes by maintaining control polygon, which allows him or her to make minute adjustments on the shape of the curve with fixed control points.

The rational Bézier curve $\gamma_{2}(t)$ listed as follows is a natural choice to meet this requirement [1].

$$
\gamma_{2}(t)=\frac{\sum_{i=0}^{n} \mathbf{P}_{i} B_{i}^{n}(t)}{\sum_{i=0}^{n} \mathbf{P}_{i} \omega_{i} B_{i}^{n}(t)}, \quad t \in[0,1] .
$$

By assigning a weight $\omega_{i}$ for each control point $\mathbf{P}_{i}$, the designer can adjust the shape of the curve by changing the value of the weights $\left\{\omega_{i}\right\}_{i=0}^{n}[2,3]$. Although the rational Bézier curve has good properties and can express the conic section, it also has disadvantages, such as difficulty in choosing the value of the weight, the increased order of rational fraction caused by the derivation, and the need for a numerical method of integration.

In addition, the algebraic trigonometric/hyperbolic curve $\gamma_{3}(t)$ with the definition domain $\alpha$ as the shape parameter is a feasible method [4-6]. Consider

$$
\gamma_{3}(t)=\sum_{i=0}^{n} \mathbf{P}_{i} u_{i}^{n}(t), \quad t \in[0, \alpha]
$$

The simple form of the algebraic trigonometric/hyperbolic curve $\gamma_{3}(t)$ can express transcendental curves (e.g., spiral and cycloid) that cannot be expressed by the Bézier curve. Nevertheless, the basis functions $\left\{u_{i}^{n}(t)\right\}_{i=0}^{n}$ include trigonometric/hyperbolic functions, such as $\sin t, \cos t, \sinh t$, and $\cosh t$. So, the algebraic trigonometric/hyperbolic curve is incompatible with the existing NURBS system, thereby restricting its application in the actual project.

In view of the fact that the expression of the parametric curve is determined by the control points and the basis functions, the properties of such functions identify the properties of the curve with its fixed control points. Therefore, several 
TABLE 1: Properties of the basis functions and the curves with shape parameters.

\begin{tabular}{|c|c|c|c|c|c|c|c|}
\hline Property & {$[7]$} & {$[8]$} & {$[9]$} & {$[10]$} & {$[11]$} & {$[12]$} & This paper \\
\hline \multicolumn{8}{|l|}{$\begin{array}{l}\text { Basis functions with multiple shape } \\
\text { parameters }\end{array}$} \\
\hline Nonnegativity & $\checkmark$ & $\checkmark$ & $\checkmark$ & $\checkmark$ & $\checkmark$ & $\checkmark$ & $\checkmark$ \\
\hline Partition of unity & $\checkmark$ & $\checkmark$ & $\checkmark$ & $\checkmark$ & $\checkmark$ & $\checkmark$ & $\checkmark$ \\
\hline Symmetry & $\checkmark$ & * & $*$ & $*$ & $\times$ & $\checkmark$ & $\checkmark$ \\
\hline Multiple shape parameters & $\times$ & $\checkmark$ & $\checkmark$ & $\checkmark$ & $\checkmark$ & $\times$ & $\checkmark$ \\
\hline Linear independence & $\checkmark$ & $\times$ & $\times$ & $\checkmark$ & $\checkmark$ & $\checkmark$ & $\checkmark$ \\
\hline Degeneracy & $\checkmark$ & $\checkmark$ & $\checkmark$ & $\times$ & $\times$ & $\checkmark$ & $\checkmark$ \\
\hline \multicolumn{8}{|l|}{ Curve with multiple shape parameters } \\
\hline Geometric and affine invariability & $\checkmark$ & $\checkmark$ & $\checkmark$ & $\checkmark$ & $\checkmark$ & $\checkmark$ & $\checkmark$ \\
\hline Convex hull property & $\checkmark$ & $\checkmark$ & $\checkmark$ & $\checkmark$ & $\checkmark$ & $\checkmark$ & $\checkmark$ \\
\hline Symmetry & $\checkmark$ & $*$ & $*$ & $*$ & $x$ & $\checkmark$ & $\checkmark$ \\
\hline Interpolation at the endpoints & $\checkmark$ & $\checkmark$ & $\checkmark$ & $\checkmark$ & $\checkmark$ & $\checkmark$ & $\checkmark$ \\
\hline Tangent at the end edge & $\checkmark$ & $\checkmark$ & $\checkmark$ & $\checkmark$ & $\times$ & $\checkmark$ & $\checkmark$ \\
\hline
\end{tabular}

${ }^{*}$ The property of symmetry in [8-10] is based on the shape parameters.

kinds of polynomial basis functions with shape parameters [7-12] and the corresponding curve have been constructed as follows.

By letting $\left\{b_{i}^{n_{1}, n_{2}}\left(t ; \lambda_{1}, \lambda_{2}, \ldots, \lambda_{m}\right)\right\}_{i=0}^{n_{1}}$ be $n_{1}+1$ polynomial functions of degree $n_{2}$ (called order $n_{1}$ and degree $n_{2}$ ) and $\left\{\mathbf{P}_{i}\right\}_{i=0}^{n_{1}}$ be $n_{1}+1$ points in spaces, the parametric curve with multiple shape parameters $\left\{\lambda_{i}\right\}_{i=0}^{m}$ is constructed as follows:

$$
\mathbf{P}\left(t ; \lambda_{1}, \lambda_{2}, \ldots, \lambda_{m}\right)=\sum_{i=0}^{n_{1}} \mathbf{P}_{i} b_{i}^{n_{1}, n_{2}}\left(t ; \lambda_{1}, \lambda_{2}, \ldots, \lambda_{m}\right) .
$$

For the sake of concision, the notations $\left\{b_{i}^{n_{1}, n_{2}}\left(t ; \lambda_{1}, \lambda_{2}, \ldots, \lambda_{m}\right)\right\}_{i=0}^{n_{1}}$ and $\mathbf{P}\left(t ; \lambda_{1}, \lambda_{2}, \ldots, \lambda_{m}\right)$ will be replaced by $\left\{b_{i}^{n_{1}, n_{2}}(t)\right\}_{i=0}^{n_{1}}$ and $\mathbf{P}(t)$. And $\left\{b_{i}^{n_{1}, n_{2}}(t)\right\}_{i=0}^{n_{1}}$ and $\mathbf{P}(t)$ will be called Quasi-Bernstein basis and Quasi-Bézier curve, respectively.

With the extra degree of freedom provided by the shape parameters $\left\{\lambda_{i}\right\}_{i=1}^{m}$ in $\left\{b_{i}^{n_{1}, n_{2}}(t)\right\}_{i=0}^{n_{1}}$, the curve $\mathbf{P}(t)$ can be freely adjusted and controlled by changing the value of $\left\{\lambda_{i}\right\}_{i=1}^{m}$ instead of changing the control points $\left\{\mathbf{P}_{i}\right\}_{i=0}^{n_{1}}$. The existing works are compared in detail in Table 1.

The construction of the basis functions with shape parameters is the key step in [7-12]. Although many kinds of basis functions with shape parameters have been obtained in the existing research, two problems need to be solved.

(1) In all existing research, the basis functions with shape parameters are initially given, and whether or not these functions and the corresponding curves have inherited the characteristics of the Bernstein basis functions and the Bézier curve, respectively, is examined. However, the method of obtaining the complex expressions of the basis functions remains unclear. Are these basis functions obtained through intuition or through an aimless attempt?

(2) There are numerous known basis functions with shape parameters in varying forms. Is there a type of Quasi-Bernstein basis function, which makes existing basis functions with shape parameters be its special case?

To answer the previous two questions, this paper uses the method of undetermined coefficients, which clarifies the construction process of the Quasi-Bernstein basis functions. A series of Quasi-Bernstein basis functions are finally obtained, rendering the existing basis function with shape parameters as their special case.

\section{Quasi-Bézier Curve}

2.1. Notation. First, the following vectors are introduced:

$$
\begin{gathered}
\mathbf{b}^{n_{1}, n_{2}}=\left(b_{0}^{n_{1}, n_{2}}(t), b_{1}^{n_{1}, n_{2}}(t), \ldots, b_{n_{1}}^{n_{1}, n_{2}}(t)\right), \\
\mathbf{P}^{n_{1}}=\left(\mathbf{P}_{0}, \mathbf{P}_{1}, \ldots, \mathbf{P}_{n_{1}}\right)^{T} .
\end{gathered}
$$

Equation (5) can be rewritten as

$$
\mathbf{P}(t)=\mathbf{b}^{n_{1}, n_{2}} \mathbf{P}^{n_{1}} .
$$


Given that $\left\{b_{i}^{n_{1}, n_{2}}(t)\right\}_{i=0}^{n_{1}}$ are polynomials with degree $n_{2}$, they can be seen as the linear combination of the Bernstein basis functions $\left\{B_{i}^{n_{2}}(t)\right\}_{i=0}^{n_{2}}$ with degree $n_{2}$ given by

$$
\begin{gathered}
\mathbf{b}^{n_{1}, n_{2}}=\mathbf{B}^{n_{2}} \mathbf{M}^{n_{2}, n_{1}}, \\
\mathbf{B}^{n_{2}}=\left(B_{0}^{n_{2}}(t), B_{1}^{n_{2}}(t), \ldots, B_{n_{2}}^{n_{2}}(t)\right), \\
\mathbf{M}^{n_{2}, n_{1}}=\left(m_{i j}\right)_{i, j=0}^{i=n_{2}, j=n_{1}} .
\end{gathered}
$$

Thus, as long as the elements in the matrix $\mathbf{M}^{n_{2}, n_{1}}$ are determined, the Quasi-Bernstein basis functions $\left\{b_{i}^{n_{1}, n_{2}}(t)\right\}_{i=0}^{n_{1}}$ with order $n_{1}$ and degree $n_{2}$ are completely constructed. Except for several elements that can be determined in $\mathbf{M}^{n_{2}, n_{1}}$, the rest are shape parameters of the Quasi-Bernstein basis functions and the Quasi-Bézier curve. Here, the matrix $\mathbf{M}^{n_{2}, n_{1}}$ is called the shape parameter matrix.

2.2. Construction of the Shape Parameter Matrix $\mathbf{M}^{n_{2}, n_{1}}$. The $\left(n_{2}+1\right)\left(n_{1}+1\right)$ elements of $m_{i j}$ in $\mathbf{M}^{n_{2}, n_{1}}$ must be determined so that $\left\{b_{i}^{n_{1}, n_{2}}(t)\right\}_{i=0}^{n_{1}}$ and $\mathbf{P}(t)$ become the Quasi-Bernstein basis functions and the Quasi-Bézier curve, respectively.

2.2.1. Determination of $m_{i j}$ according to the Characteristics of the Quasi-Bernstein Basis Functions. The Quasi-Bernstein basis functions $\left\{b_{i}^{n_{1}, n_{2}}(t)\right\}_{i=0}^{n_{1}}$ with order $n_{1}$ and degree $n_{2}$ must satisfy the characteristics of nonnegativity, normalization, symmetry, linear independence, and degeneracy.

Proposition 1 (nonnegativity). A sufficient condition for $b_{i}^{n_{1}, n_{2}}(t) \geq 0\left(i=0,1, \ldots, n_{2}\right)$ is

$$
m_{i j} \geq 0 \quad\left(i=0,1, \ldots, n_{2}, j=0,1, \ldots, n_{1}\right) .
$$

Proof. Here, $b_{j}^{n_{1}, n_{2}}(t)=\sum_{i=0}^{n_{2}} m_{i j} B_{i}^{n_{2}}(t)$ is known to have been extracted from (8). Based on the non-negativity of the Bernstein basis functions $\left\{B_{i}^{n_{2}}(t)\right\}_{i=0}^{n_{2}}$, a sufficient condition for the non-negativity of the Quasi-Bernstein basis functions $\left\{b_{i}^{n_{1}, n_{2}}(t)\right\}_{i=0}^{n_{1}}$ is the non-negativity of the elements $m_{i j}$ in $\mathbf{M}^{n_{2}, n_{1}}$. Hence, $m_{i j}$ must satisfy (9).

Note 1. Clearly, there is no row with all elements being 0 in $\mathbf{M}^{n_{2}, n_{1}}$. In other words,

$$
\sum_{i=0}^{n_{2}} m_{i j} \neq 0 \quad\left(j=0,1 \ldots, n_{1}\right) .
$$

Proposition 2 (normalization). The necessary and sufficient condition for $\sum_{j=0}^{n_{1}} b_{j}^{n_{1}, n_{2}}(t)=1$ is given by

$$
\sum_{j=0}^{n_{1}} m_{i j}=1 \quad\left(i=0,1, \ldots, n_{2}\right) .
$$

Proof. It is known that

$$
\begin{aligned}
\sum_{j=0}^{n_{1}} b_{j}^{n_{1}, n_{2}}(t)-1 \\
=\sum_{j=0}^{n_{1}}\left(\sum_{i=0}^{n_{2}} m_{i j} B_{i}^{n_{2}}(t)\right)-1 \\
=\sum_{i=0}^{n_{2}}\left(\sum_{j=0}^{n_{1}} m_{i j}\right) B_{i}^{n_{2}}(t)-1 \\
=\sum_{i=0}^{n_{2}}\left(\sum_{j=0}^{n_{1}} m_{i j}\right) B_{i}^{n_{2}}(t)-\sum_{i=0}^{n_{2}} B_{i}^{n_{2}}(t) \\
=\sum_{i=0}^{n_{2}}\left(\sum_{j=0}^{n_{1}} m_{i j}-1\right) B_{i}^{n_{2}}(t) .
\end{aligned}
$$

According to the linear independence of the Bernstein basis functions $\left\{B_{i}^{n_{2}}(t)\right\}_{i=0}^{n_{2}}$, the necessary and sufficient condition for $\sum_{j=0}^{n_{1}} b_{j}^{n_{1}, n_{2}}(t)-1=0$ is $\sum_{j=0}^{n_{1}} m_{i j}=1(i=$ $\left.0,1, \ldots, n_{2}\right)$.

Note 2. By combining (9) and (11), $m_{i j}$ satisfies $0 \leq m_{i j} \leq$ $1\left(i=0,1, \ldots, n_{2}, j=0,1, \ldots, n_{1}\right)$.

Proposition 3 (symmetry). The necessary and sufficient condition for $b_{j}^{n_{1}, n_{2}}(t)=b_{n_{1}-j}^{n_{1}, n_{2}}(1-t)\left(j=0,1, \ldots, n_{1}\right)$ is given by

$$
m_{i j}=m_{n_{2}-i, n_{1}-j} \quad\left(i=0,1, \ldots, n_{2}, j=0,1, \ldots, n_{1}\right) .
$$

Proof. According to the symmetry of the Bernstein basis functions $\left\{B_{i}^{n_{2}}(t)\right\}_{i=0}^{n_{2}}$ of $B_{i}^{n_{2}}(t)=B_{n_{2}-i}^{n_{2}}(1-t)$, the following can be derived:

$$
\begin{aligned}
& b_{n_{1}-j}^{n_{1}, n_{2}}(1-t)-b_{j}^{n_{1}, n_{2}}(t) \\
& =\sum_{i=0}^{n_{2}} B_{i}^{n_{2}}(1-t) m_{i, n_{1}-j}-\sum_{i=0}^{n_{2}} B_{i}^{n_{2}}(t) m_{i j} \\
& =\sum_{i=0}^{n_{2}} B_{n_{2}-i}^{n_{2}}(t) m_{i, n_{1}-j}-\sum_{i=0}^{n_{2}} B_{i}^{n_{2}}(t) m_{i j} \\
& =\sum_{i=0}^{n_{2}} B_{i}^{n_{2}}(t) m_{n_{2}-i, n_{1}-j}-\sum_{i=0}^{n_{2}} B_{i}^{n_{2}}(t) m_{i j} \\
& =\sum_{i=0}^{n_{2}} B_{i}^{n_{2}}(t)\left(m_{n_{2}-i, n_{1}-j}-m_{i j}\right), \\
& j=0,1, \ldots, n_{1} .
\end{aligned}
$$

According to the linear independence of the Bernstein basis functions $\left\{B_{i}^{n_{2}}(t)\right\}_{i=0}^{n_{2}}$, the necessary and sufficient condition for $b_{j}^{n_{1}, n_{2}}(t)-b_{n_{1}-j}^{n_{1}, n_{2}}(1-t)=0$ is (13). 
Proposition 4 (linear independence). The necessary and sufficient condition for the linear independence of $\left\{b_{i}^{n_{1}, n_{2}}(t)\right\}_{i=0}^{n_{1}}$ is given by

$$
r\left(\mathbf{M}^{n_{2}, n_{1}}\right)=n_{1}+1
$$

Proof. It is known that

$$
\begin{aligned}
\sum_{j=0}^{n_{1}} k_{j} b_{j}^{n_{1}, n_{2}}(t) & =\sum_{j=0}^{n_{1}} k_{j}\left(\sum_{i=0}^{n_{2}} m_{i j} B_{i}^{n_{2}}(t)\right) \\
& =\sum_{i=0}^{n_{2}}\left(\sum_{j=0}^{n_{1}} k_{j} m_{i j}\right) B_{i}^{n_{2}}(t) .
\end{aligned}
$$

According to the linear independence of the Bernstein basis functions $\left\{B_{i}^{n_{2}}(t)\right\}_{i=0}^{n_{2}}$, the necessary and sufficient condition for $\sum_{j=0}^{n_{1}} k_{j} b_{j}^{n_{1}, n_{2}}(t)=0$ is given by

$$
\sum_{j=0}^{n_{1}} k_{j} m_{i j}=0 \quad\left(i=0,1, \ldots, n_{2}\right) .
$$

$\mathbf{M}^{j}=\left(m_{0, j}, m_{1, j}, \ldots, m_{n_{2}, j}\right)^{T}$ is defined as the $j$ th column vector of $\mathbf{M}^{n_{2}, n_{1}}$. Equation (17) is equivalent to $\sum_{j=0}^{n_{1}} k_{j} \mathbf{M}^{j}=0$. Thus, the necessary and sufficient condition for the linear independence of $\left\{b_{i}^{n_{1}, n_{2}}(t)\right\}_{i=0}^{n_{1}}$ is also the linear independence of the column vectors $\left\{\mathbf{M}^{j}\right\}_{i=0}^{n_{1}}$ of the matrix $\mathbf{M}^{n_{2}, n_{1}}$. Consequently, the necessary and sufficient condition for the linear independence of $\left\{b_{i}^{n_{1}, n_{2}}(t)\right\}_{i=0}^{n_{1}}$ is that the rank of the shape parameter matrix $\mathbf{M}^{n_{2}, n_{1}}$ satisfies $r\left(\mathbf{M}^{n_{2}, n_{1}}\right)=n_{1}+1$.

Note 3. When (15) is true, $n_{2} \geq n_{1}$.

Proposition 5 (degeneracy). If the elements $\left\{m_{i j}\right\}_{i, j=0}^{i=n_{2}, j=n_{1}}$ in the matrix $\mathbf{M}^{n_{2}, n_{1}}$ are represented by (18), the Quasi-Bernstein basis functions $\left\{b_{i}^{n_{1}, n_{2}}(t)\right\}_{i=0}^{n_{1}}$ with order $n_{1}$ and degree $n_{2}$ are degenerated into the Bernstein basis functions $\left\{B_{i}^{n_{1}}(t)\right\}_{i=0}^{n_{1}}$ with $\operatorname{order} n_{1}$.

$$
\begin{aligned}
& m_{i j} \\
& = \begin{cases}\frac{\left(\begin{array}{c}
n_{2}-n_{1} \\
i-j
\end{array}\right)\left(\begin{array}{c}
n_{1} \\
j
\end{array}\right)}{\left(\begin{array}{c}
n_{2} \\
i
\end{array}\right)}, & \max \left(0, i-\left(n_{2}-n_{1}\right)\right) \leq j \leq \min \left(n_{1}, i\right), \\
0, & 0 \leq i \leq n_{2}, 0 \leq j \leq n_{1} ; \\
\text { else. }\end{cases}
\end{aligned}
$$

Proof. When the elements $\left\{m_{i j}\right\}_{i, j=0}^{i=n_{2}, j=n_{1}}$ in the matrix $\mathbf{M}^{n_{2}, n_{1}}$ are represented by (18), the following is obtained:

$$
\mathbf{B}^{n_{1}}=\mathbf{B}^{n_{2}} \mathbf{M}^{n_{2}, n_{1}}
$$

Comparing (19) with (8), Proposition 5 is proven.

Note 4. If $n_{1}=n_{2}, \mathbf{M}^{n_{2}, n_{1}}$ is an identity matrix here.

\subsubsection{Determination of $m_{i j}$ according to the Characteristics of the Quasi-Bézier Curve}

Proposition 6 (interpolation at the endpoints). The necessary and sufficient condition for $\mathbf{P}(0)=\mathbf{P}_{0}$ and $\mathbf{P}(1)=\mathbf{P}_{n_{1}}$ is given by

$$
\begin{array}{r}
m_{00}=1, \quad m_{0 j}=0 \quad\left(j=1,2, \ldots, n_{1}\right), \\
m_{n_{2}, n_{1}}=1, \quad m_{n_{2}, j}=0 \quad\left(j=0,1, \ldots, n_{1}-1\right) .
\end{array}
$$

Proof. Clearly, the necessary and sufficient condition for $\mathbf{P}(0)=\mathbf{P}_{0}$ is the Quasi-Bernstein primary functions $\left\{b_{i}^{n_{1}, n_{2}}(t)\right\}_{i=0}^{n_{1}}$ satisfying the following:

$$
b_{i}^{n_{1}, n_{2}}(0)= \begin{cases}1, & i=0 \\ 0, & i \neq 0\end{cases}
$$

It is known that

$$
\begin{aligned}
\left(b_{0}^{n_{1}, n_{2}}\right. & \left.(0), b_{1}^{n_{1}, n_{2}}(0), \ldots, b_{n_{1}}^{n_{1}, n_{2}}(0)\right) \\
& =\left(B_{0}^{n_{2}}(0), B_{1}^{n_{2}}(0), \ldots, B_{n_{2}}^{n_{2}}(0)\right) \cdot\left(m_{i j}\right)_{i, j=0}^{i=n_{2}, j=n_{1}} \\
& =(1,0, \ldots, 0) \cdot\left(m_{i j}\right)_{i, j=0}^{i=n_{2}, j=n_{1}} \\
& =\left(m_{00}, m_{01}, \ldots, m_{0, n_{1}-1}, m_{0 n_{1}}\right) .
\end{aligned}
$$

Thus, the necessary and sufficient condition for $\left(b_{0}^{n_{1}, n_{2}}(0)\right.$, $\left.b_{1}^{n_{1}, n_{2}}(0), \ldots, b_{n_{1}}^{n_{1}, n_{2}}(0)\right)=(1,0, \ldots, 0)$ is $(20)$.

Similarly, the necessary and sufficient condition for $\mathbf{P}(1)=\mathbf{P}_{n_{1}}$ is the Quasi-Bernstein primary functions $\left\{b_{i}^{n_{1}, n_{2}}(t)\right\}_{i=0}^{n_{1}}$ satisfying the following:

$$
b_{i}^{n_{1}, n_{2}}(1)= \begin{cases}1, & i=n_{1}, \\ 0, & i \neq n_{1} .\end{cases}
$$

It is known that

$$
\begin{aligned}
\left(b_{0}^{n_{1}, n_{2}}\right. & \left.(1), b_{1}^{n_{1}, n_{2}}(1), \ldots, b_{n_{1}}^{n_{1}, n_{2}}(1)\right) \\
& =\left(B_{0}^{n_{2}}(1), B_{1}^{n_{2}}(1), \ldots, B_{n_{2}}^{n_{2}}(1)\right) \cdot\left(m_{i j}\right)_{i, j=0}^{i=n_{2}, j=n_{1}} \\
& =(0, \ldots, 0,1) \cdot\left(m_{i j}\right)_{i, j=0}^{i=n_{2}, j=n_{1}} \\
& =\left(m_{n_{2}, 0}, m_{n_{2}, 1}, \ldots, m_{n_{2}, n_{1}-1}, m_{n_{2}, n_{1}}\right) .
\end{aligned}
$$

Thus, the necessary and sufficient condition for $\left(b_{0}^{n_{1}, n_{2}}(1), b_{1}^{n_{1}, n_{2}}(1), \ldots, b_{n_{1}}^{n_{1}, n_{2}}(1)\right)=(0, \ldots, 0,1)$ is $(21)$.

Hence, the necessary and sufficient condition for $\mathbf{P}(1)=$ $\mathbf{P}_{n_{1}}$ is (21).

Note 5. When $\left\{b_{i}^{n_{1}, n_{2}}(t)\right\}_{i=0}^{n_{1}}$ have the property of symmetry, (21) is equivalent to (20) according to (13). 
Proposition 7 (tangent edges at the endpoints). The necessary and sufficient condition for $\mathbf{P}^{\prime}(0)\left\|\mathbf{P}_{1} \mathbf{P}_{0}, \mathbf{P}^{\prime}(1)\right\| \mathbf{P}_{n} \mathbf{P}_{n-1}$ is given by

$$
\begin{gathered}
m_{10}+m_{11}=1, \quad m_{10} \neq 1, \\
m_{1 j}=0 \quad\left(j=2,3, \ldots, n_{1}\right), \\
m_{n_{2}-1, n_{1}-1}+m_{n_{2}-1, n_{1}}=1, \quad m_{n_{2}-1, n_{1}} \neq 1, \\
m_{n_{2}-1, j}=0 \quad\left(j=0,1, \ldots, n_{1}-2\right) .
\end{gathered}
$$

Proof. It is known that

$$
\begin{aligned}
\mathbf{P}^{\prime}(0)= & \left.\mathbf{P}^{\prime}(t)\right|_{t=0} \\
= & \left.\left(b_{0}^{n_{1}, n_{2}}(t), b_{1}^{n_{1}, n_{2}}(t), \ldots, b_{n_{1}}^{n_{1}, n_{2}}(t)\right)^{\prime}\right|_{t=0} \\
& \times\left(\mathbf{P}_{0}, \mathbf{P}_{1}, \ldots, \mathbf{P}_{n_{1}}\right)^{T} \\
= & \left.\left(B_{0}^{n_{2}}(t), B_{1}^{n_{2}}(t), \ldots, B_{n_{2}}^{n_{2}}(t)\right)^{\prime}\right|_{t=0} \cdot\left(m_{i j}\right)_{i, j=0}^{i=n_{2}, j=n_{1}} \\
& \cdot\left(\mathbf{P}_{0}, \mathbf{P}_{1}, \ldots, \mathbf{P}_{n_{1}}\right)^{T} \\
= & n_{2}(-1,1,0, \ldots, 0) \cdot\left(m_{i j}\right)_{i, j=0}^{i=n_{2}, j=n_{1}} \cdot\left(\mathbf{P}_{0}, \mathbf{P}_{1}, \ldots, \mathbf{P}_{n_{1}}\right)^{T} \\
= & n_{2}\left(m_{10}-m_{00}, m_{11}-m_{01}, \ldots, m_{1 n_{1}}-m_{0 n_{1}}\right) \\
& \times\left(\mathbf{P}_{0}, \mathbf{P}_{1}, \ldots, \mathbf{P}_{n_{1}}\right)^{T} .
\end{aligned}
$$

Clearly, the necessary and sufficient condition for $\mathbf{P}^{\prime}(0) \| \mathbf{P}_{1} \mathbf{P}_{0}$ is $\left(m_{10}-m_{00}\right) /\left(m_{11}-m_{01}\right)=-1, m_{1 j}-m_{0 j}=$ $0\left(j=2,3, \ldots, n_{1}\right)$ which verifies $(26)$.

Similarly, the necessary and sufficient condition for $\mathbf{P}^{\prime}(1) \| \mathbf{P}_{n} \mathbf{P}_{n-1}$ is (27).

Note 6. When $\left\{b_{i}^{n_{1}, n_{2}}(t)\right\}_{i=0}^{n_{1}}$ have the property of symmetry, (27) is equivalent to (26) according to (13).

2.2.3. Form of Shape Parameter Matrix $\mathbf{M}^{n_{2}, n_{1}}$. All shape parameter matrixes that satisfy (9), (11), (13), (15), (20), (21), (26), and (27) have the following form:

$$
\mathbf{M}^{n_{2}, n_{1}}=\left(\begin{array}{ccccc}
1 & 0 & \cdots & 0 & 0 \\
m_{10} & 1-m_{10} & \cdots & 0 & 0 \\
m_{20} & m_{21} & \cdots & m_{2, n_{1}-1} & m_{2 n_{1}} \\
\vdots & \vdots & \ddots & \vdots & \vdots \\
m_{2 n_{1}} & m_{2, n_{1}-1} & \cdots & m_{21} & m_{20} \\
0 & 0 & \cdots & 1-m_{10} & m_{10} \\
0 & 0 & \cdots & 0 & 1
\end{array}\right)_{\left(n_{2}+1\right) \times\left(n_{1}+1\right)}
$$

Here, $m_{i j}$ are variable shape parameters that satisfy

$$
\begin{aligned}
& \sum_{j=0}^{n_{1}} m_{i j}=1 \quad\left(i=2,3, \ldots,\left[\frac{\left(n_{2}+1\right)}{2}\right]\right), 0 \leq m_{10}<1, \\
& 0 \leq m_{i j} \leq 1\left(i=2,3, \ldots,\left[\frac{\left(n_{2}+1\right)}{2}\right], j=0,1, \ldots, n_{1}\right) .
\end{aligned}
$$

2.3. The Characteristics of the Quasi-Bézier Curve. In summary, the Quasi-Bézier curve $\mathbf{P}(t)$ based on the QuasiBernstein basis functions $\left\{b_{i}^{n_{1}, n_{2}}(t)\right\}_{i=0}^{n_{1}}$ has the characteristics listed as follows:

(a) shape adjustability: the shape of the Quasi-Bézier curve can still be adjusted by maintaining the control points.

(b) geometric invariability: the Quasi-Bézier curve only relies on the control points, whereas it has nothing to do with the position and direction of the coordinate system; in other words, the curve shape remains invariable after translation and revolving in the coordinate system;

(c) affine invariability: barycentric combinations are invariant under affine maps; therefore, (9) and (11) give the algebraic verification of this property;

(d) symmetry: whether the control points are labeled $\mathbf{P}_{0} \mathbf{P}_{1} \cdots \mathbf{P}_{n_{1}}$ or $\mathbf{P}_{n_{1}} \mathbf{P}_{n_{1}-1} \cdots \mathbf{P}_{0}$, the curves that correspond to the two different orderings look the same; they differ only in the direction in which they are traversed, and this is written as

$$
\sum_{i=0}^{n_{1}} \mathbf{P}_{i} b_{i}^{n_{1}, n_{2}}(t)=\sum_{i=0}^{n_{1}} \mathbf{P}_{n_{1}-i} b_{n_{1}-i}^{n_{1}, n_{2}}(1-t),
$$

which follows the inspection of (13);

(e) convex hull property: this property exists since the Quasi-Bernstein basis functions $\left\{b_{i}^{n_{1}, n_{2}}(t)\right\}_{i=0}^{n_{1}}$ have the properties of non-negativity and normalization; the Quasi-Bézier curve is the convex linear combination of control points, and as such, it is located in the convex hull of the control points;

(f) interpolation at the endpoints and tangent edges at the endpoint: the Quasi-Bézier curve $\mathbf{P}(t)$ interpolates the first and the last control points $\mathbf{P}(0)=$ $\mathbf{P}_{0}$ and $\mathbf{P}(1)=\mathbf{P}_{n_{1}}$; the first and last edges of the control polygon are the tangent lines at the endpoints, where $\mathbf{P}^{\prime}(0) \| \mathbf{P}_{1} \mathbf{P}_{0}$ and $\mathbf{P}^{\prime}(1) \| \mathbf{P}_{n} \mathbf{P}_{n-1}$.

2.4. Geometric Significance of the Shape Parameters. According to (29), when $m_{i, j_{0}}\left(i=0,1, \ldots, n_{2}, j_{0}=0,1, \ldots, n_{1}\right)$ increases, $b_{j_{0}}^{n_{1}, n_{2}}(t)$ and $b_{n_{1}-j_{0}}^{n_{1}, n_{2}}(t)$ increase as well; specifically, $\mathbf{P}(t)$ comes close to the control points $\mathbf{P}_{j_{0}}$ and $\mathbf{P}_{n_{1}-j_{0}}$. The geometric significance of the shape parameters is shown in Section 3. 


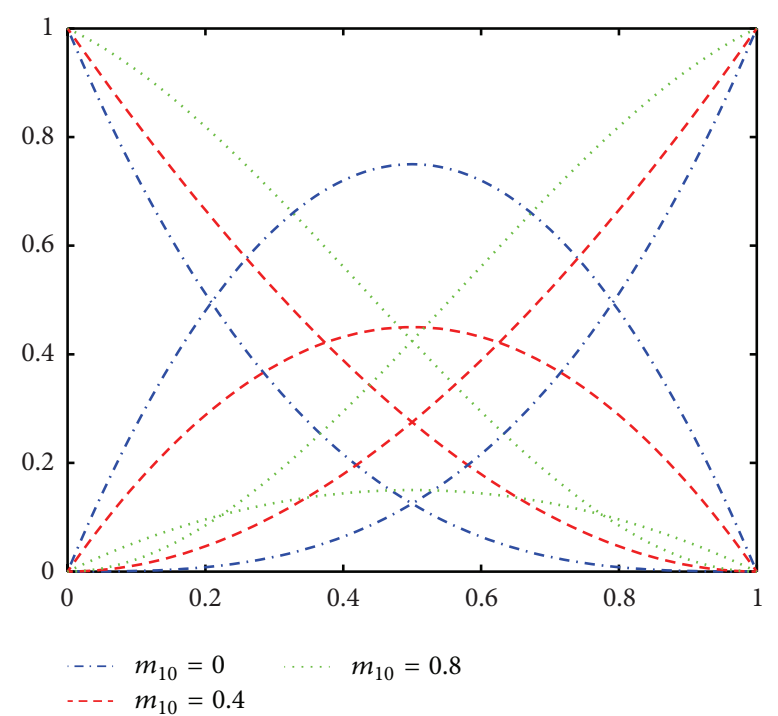

(a)

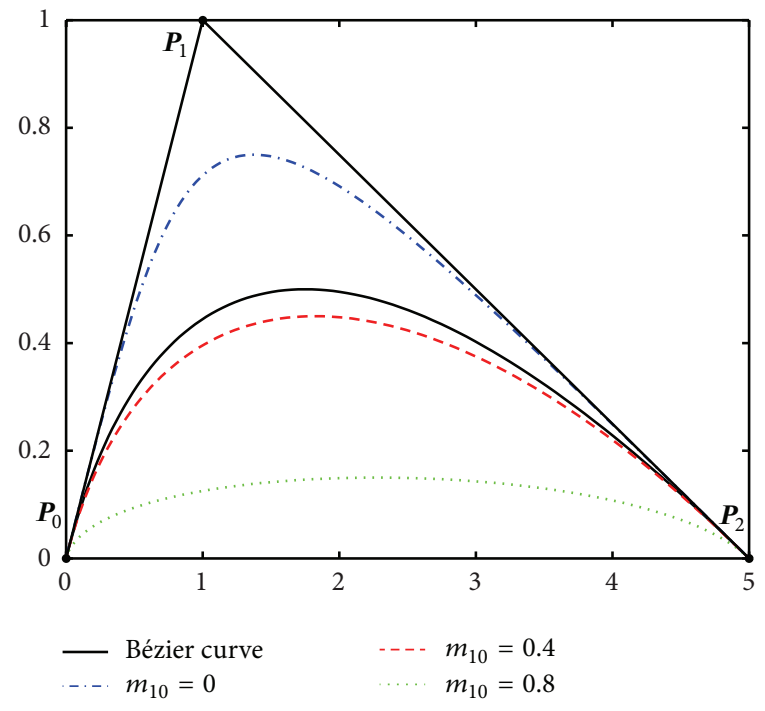

(b)

Figure 1: Quasi-Bernstein basis functions and Quasi-Bézier curves when $n_{1}=2$ and $n_{2}=3$.

\section{Numerical Examples}

Example 1. The shape parameter matrix $\mathbf{M}^{3,2}$ is constructed from (29). The corresponding Quasi-Bernstein basis functions and the Quasi-Bézier curves with different shape parameter $m_{10}$ are given as follows:

$$
\mathbf{M}^{3,2}=\left(\begin{array}{ccc}
1 & 0 & 0 \\
m_{10} & 1-m_{10} & 0 \\
0 & 1-m_{10} & m_{10} \\
0 & 0 & 1
\end{array}\right), \quad 0 \leq m_{10}<1 .
$$

The geometric significance of the shape parameters $m_{10}$ is shown in Figure 1. As the value of the shape parameter $m_{10}$ increases, the elements in the second column of $\mathbf{M}^{3,2}$ decrease. According to (8), the second Quasi-Bernstein basis function $b_{1}^{2,3}(t)$ decreases. So, the corresponding QuasiBézier curve moves away from the control point $\mathbf{P}_{1}$ (see Figure 1(b)).

Example 2. The shape parameter matrix $\mathbf{M}^{4,2}$ is constructed from (29). The corresponding Quasi-Bernstein basis functions and the Quasi-Bézier curves with different shape parameters $m_{10}$ and $m_{20}$ are given as follows:

$$
\begin{gathered}
\mathbf{M}^{4,2}=\left(\begin{array}{ccc}
1 & 0 & 0 \\
m_{10} & 1-m_{10} & 0 \\
m_{20} & 1-2 m_{20} & m_{20} \\
0 & 1-m_{10} & m_{10} \\
0 & 0 & 1
\end{array}\right), \\
0 \leq m_{10}<1,0 \leq m_{20} \leq \frac{1}{2} .
\end{gathered}
$$

The geometric significance of the shape parameters $m_{10}$ and $m_{20}$ is shown in Figure 2. When we increase the value of $m_{10}$ and keep $m_{20}$ unchanged, the elements in the second column of $\mathbf{M}^{4,2}$ decrease. According to (8), the second QuasiBernstein basis function $b_{1}^{2,4}(t)$ decreases. Compare the blue curve with the red one, and we will find that the Quasi-Bézier curve moves away from the control point $\mathbf{P}_{1}$ (see Figure $2(\mathrm{~b})$ ).

If we increase the value of $m_{20}$ and keep $m_{10}$ unchanged, similar result is also obtained. Compare the red curve with the green one, and we will find that the Quasi-Bézier curve moves away from the control point $\mathbf{P}_{1}$ (see Figure 2(b)).

Example 3. The shape parameter matrix $\mathbf{M}^{3,3}$ is constructed from (29). The corresponding Quasi-Bernstein basis functions and the Quasi-Bézier curves with different shape parameter $m_{10}$ are given as follows:

$$
\mathbf{M}^{3,3}=\left(\begin{array}{cccc}
1 & 0 & 0 & 0 \\
m_{10} & 1-m_{10} & 0 & 0 \\
0 & 0 & 1-m_{10} & m_{10} \\
0 & 0 & 0 & 1
\end{array}\right), \quad 0 \leq m_{10}<1
$$

The geometric significance of the shape parameters $m_{10}$ is shown in Figure 3. As the value of the shape parameter $m_{10}$ increases, the elements in the second and the third column of $\mathbf{M}^{3,3}$ decrease. According to (8), the second Quasi-Bernstein basis function $b_{1}^{3,3}(t)$ and the third Quasi-Bernstein basis function $b_{2}^{3,3}(t)$ decrease. So, the corresponding Quasi-Bézier curve moves away from the control points $\mathbf{P}_{1}$ and $\mathbf{P}_{2}$ (see Figure 3(b)).

Example 4. The shape parameter matrix $\mathbf{M}^{4,3}$ is constructed from (29). The corresponding Quasi-Bernstein basis 


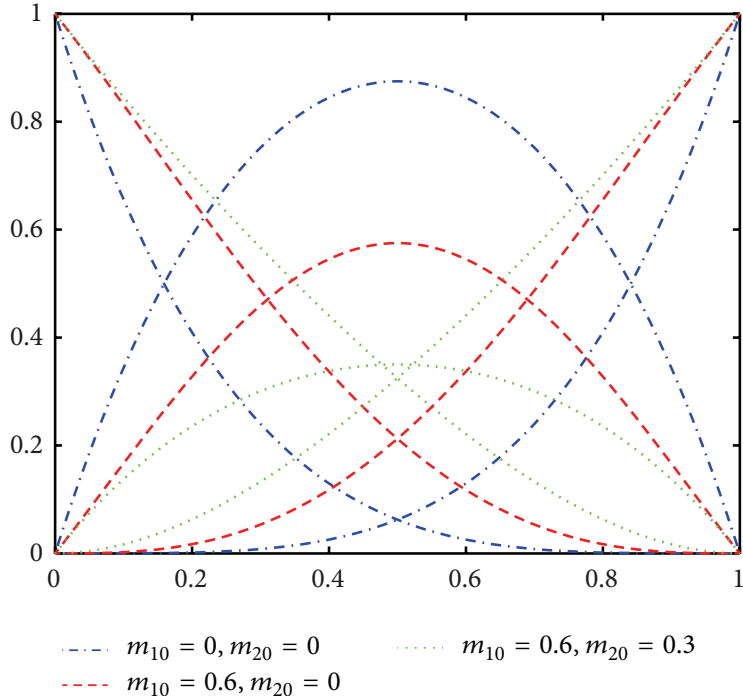

(a)

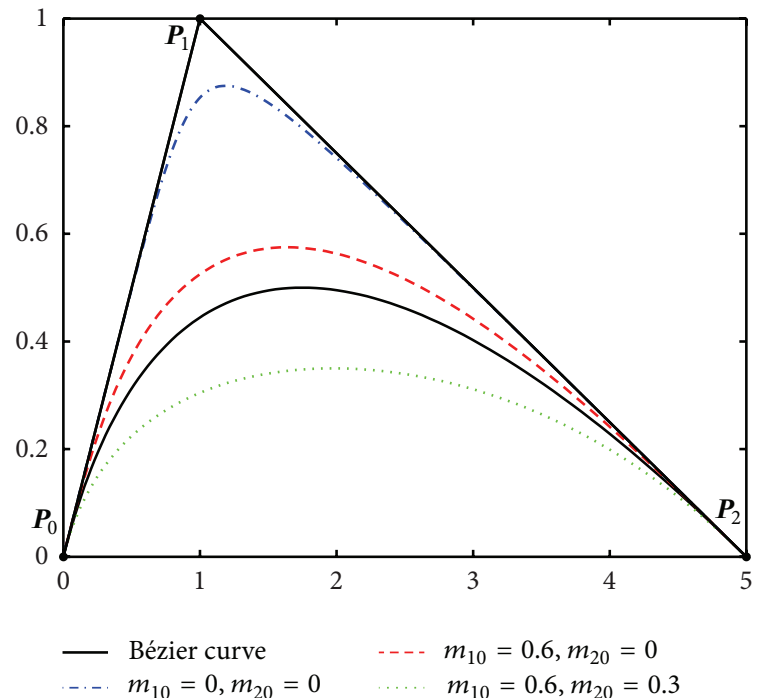

(b)

FIgURE 2: Quasi-Bernstein basis functions and Quasi-Bézier curves when $n_{1}=2$ and $n_{2}=4$.

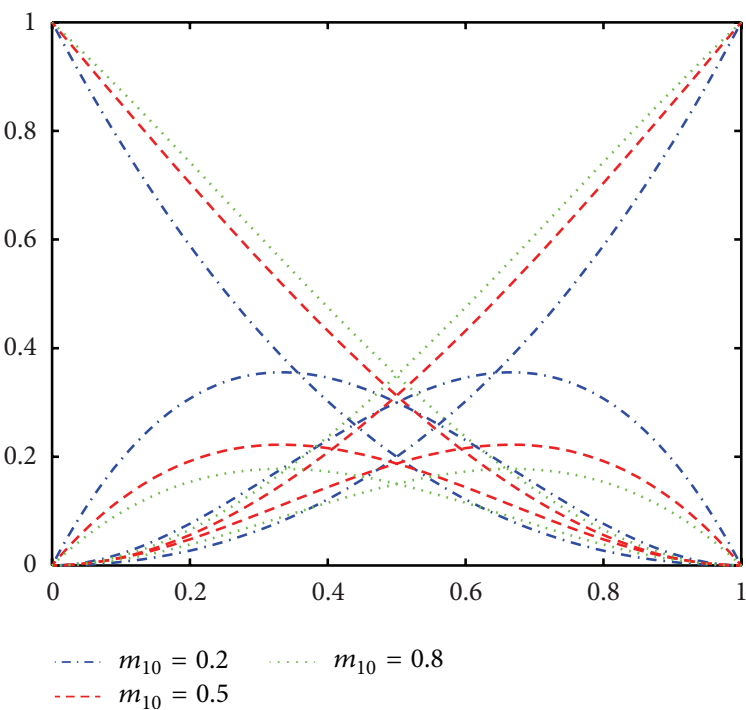

(a)

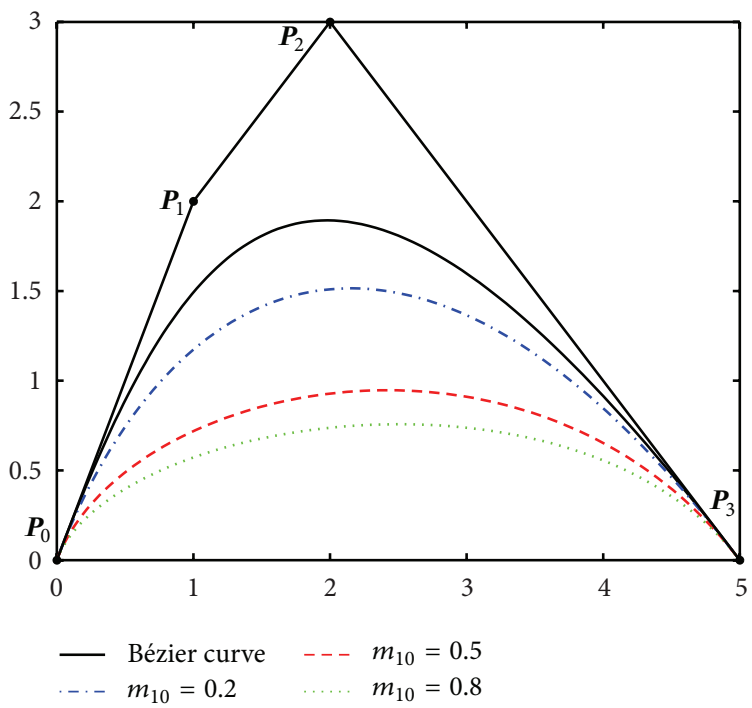

(b)

FIgURE 3: Quasi-Bernstein basis functions and Quasi-Bézier curves when $n_{1}=3$ and $n_{2}=3$.

functions and the Quasi-Bézier curves with different shape parameter $m_{10}$ and $m_{20}$ are given as follows:

$$
\mathbf{M}^{4,3}=\left(\begin{array}{cccc}
1 & 0 & 0 & 0 \\
m_{10} & 1-m_{10} & 0 & 0 \\
m_{20} & \frac{1}{2}-m_{20} & \frac{1}{2}-m_{20} & m_{20} \\
0 & 0 & 1-m_{10} & m_{10} \\
0 & 0 & 0 & 1
\end{array}\right),
$$

The geometric significance of the shape parameters $m_{10}$ and $m_{20}$ is shown in Figure 4. When we increase the value of $m_{10}$ and keep $m_{20}$ unchanged, the elements in the second and the third column of $\mathbf{M}^{4,3}$ decrease. According to (8), the second Quasi-Bernstein basis function $b_{1}^{3,4}(t)$ and the third Quasi-Bernstein basis function $b_{2}^{3,4}(t)$ decrease. Compare the blue curve with the red one, and we will find that the QuasiBézier curve moves away from the control points $\mathbf{P}_{1}$ and $\mathbf{P}_{2}$ (see Figure 4(b)).

If we increase the value of $m_{20}$ and keep $m_{10}$ unchanged, similar result is also obtained. Compare the red curve with the green one, and we will find that the Quasi-Bézier curve moves away from the control points $\mathbf{P}_{1}$ and $\mathbf{P}_{2}$ (see Figure 4(b)). 


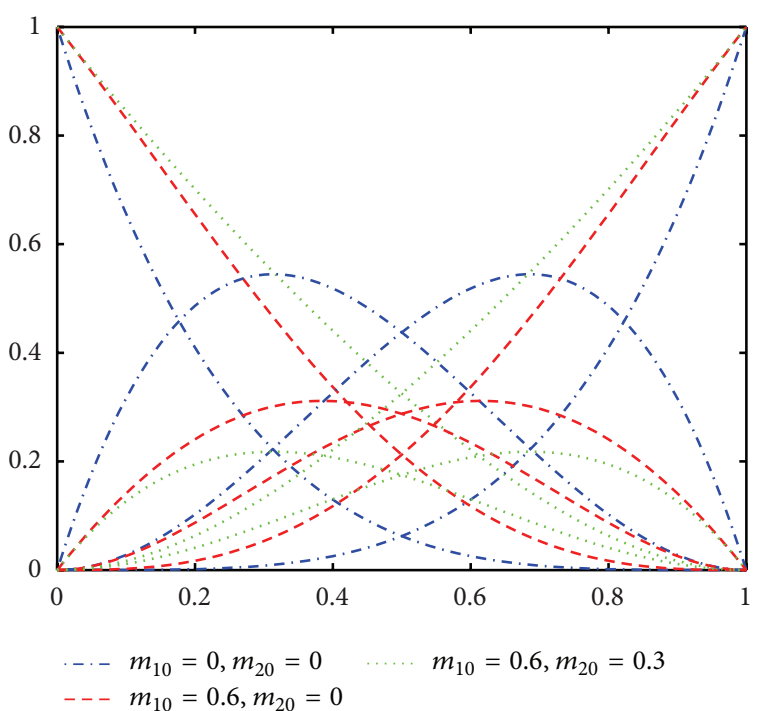

(a)

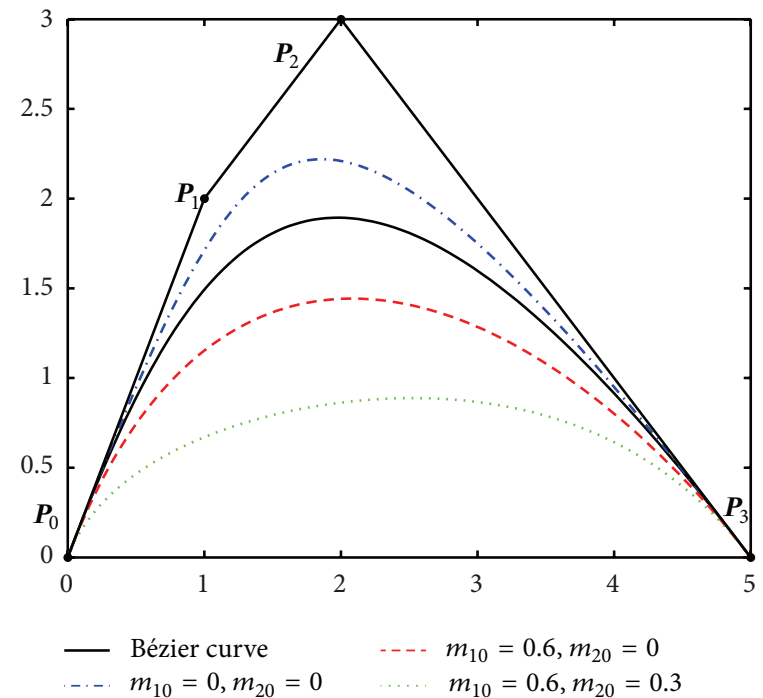

(b)

FIgURe 4: Quasi-Bernstein basis functions and Quasi-Bézier curves when $n_{1}=3$ and $n_{2}=4$.

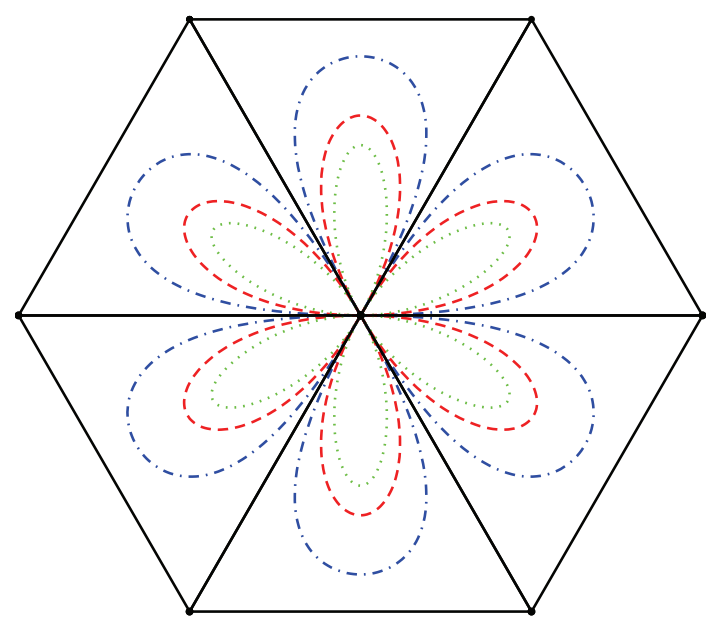

FIgURE 5: Three kinds of flowers with six petals.

Note 7. Several Quasi-Bernstein basis functions for low degree and low order are presented aforementioned. The corresponding basis functions for higher degree and higher order are defined recursively as follows [7, 12]:

$$
\begin{array}{r}
b_{i}^{n_{1}+1, n_{2}+1}(t)=(1-t) b_{i}^{n_{1}, n_{2}}(t)+t b_{i-1}^{n_{1}, n_{2}}(t), \\
i=0,1, \ldots, n_{1} .
\end{array}
$$

Here, we set $b_{-1}^{n_{1}, n_{2}}(t)=b_{n_{1}+1}^{n_{1}, n_{2}}(t)=0$.

Example 5. Figure 5 presents three kinds of flowers with six petals, defined by six symmetric control polygons. Similar flowers are obtained from the same control polygons with different shape parameters.

Example 6. Figure 6 presents three kinds of outlines of the vase, all of which are similar to the control polygons. So, the

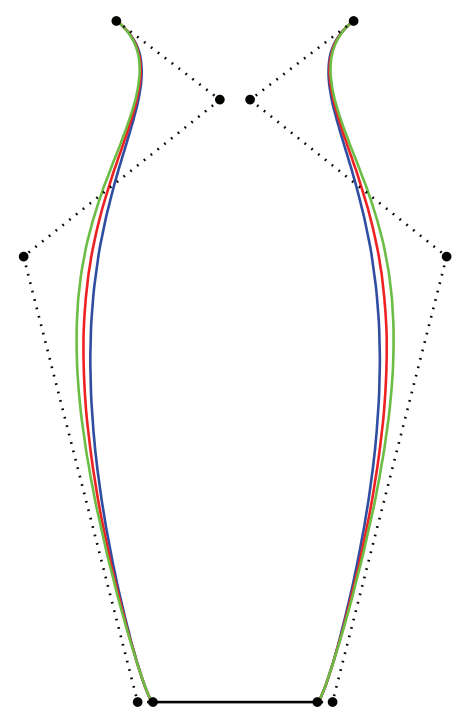

FIgURE 6: Three kinds of outlines of the vase.

designer can make minute adjustments with the same control polygons by changing the value of the shape parameters.

\section{Discussion}

4.1. Special Cases. Several existing basis functions containing just one shape parameter in $[7,12]$ are considered as the special cases in this paper. Meanwhile, for the polynomial basis functions with multiple shape parameters in [8-11], the symmetry was not discussed by authors. In fact, when these shape parameters satisfy certain relations, the corresponding basis functions and curves become symmetrical. Then, the curves have geometric and affine invariability, convex hull property, symmetry, interpolation at the endpoints, and 
tangent edges at the endpoints, and the corresponding shape parameter matrices are the special cases of (29).

We take [9] as example. When the shape parameters satisfy certain relations in [9], the shape parameter matrix is

$$
\begin{aligned}
& \mathbf{M}^{n+1, n} \\
& =\left(\begin{array}{ccccc}
1 & 0 & \cdots & 0 & 0 \\
\frac{1-\lambda_{1}}{n+1} & 1-\frac{1-\lambda_{1}}{n+1} & \cdots & 0 & 0 \\
0 & \frac{2-\lambda_{2}}{n+1} & \cdots & 0 & 0 \\
\vdots & \vdots & \ddots & \vdots & \vdots \\
0 & 0 & \cdots & \frac{2-\lambda_{2}}{n+1} & 0 \\
0 & 0 & \cdots & 1-\frac{1-\lambda_{1}}{n+1} & \frac{1-\lambda_{1}}{n+1} \\
0 & 0 & \cdots & 0 & 1
\end{array}\right)_{(n+2) \times(n+1)} .
\end{aligned}
$$

It is the special case of (29).

4.2. Degree and Order of the Curve. In the previous work, the difference between the degree and order of the curve is fixed (i.e., $n_{2}-n_{1}=0$ in $[10,11], n_{2}-n_{1}=1$ in [7-9], and $n_{2}-n_{1}=2$ in [12]), and the scope of the curve is also fixed with the same control points.

However, comparing Figures 1(a) and 1(b), it is found that the greater the difference between degree and order, the larger the scope of the Quasi-Bézier curve acquired. In order to obtain the Quasi-Bézier curves with broader scope with the same control points, the designer can increase the difference between the degree and the order $n_{2}-n_{1}$.

\section{Conclusion and Further Work}

In this paper, a series of univariate Quasi-Bernstein basis functions are constructed, thereby creating a series of QuasiBézier curves. The shape of the series of curves can be adjusted even with the control points fixed. The Quasi-Bézier curves also possess geometric and affine invariability, convex hull property, symmetry, interpolation at the endpoints, and tangent edges at the endpoints.

Quasi-Bernstein basis functions with shape parameters have been directly studied in the previous research. However, in this paper, each function has been gradually inferred and constructed using a clear method of undetermined coefficients, where each shape parameter is proposed according to the properties of the Quasi-Bernstein basis functions and the Quasi-Bézier curve. Under the premise of satisfying symmetry, the former basis functions are all considered as the special cases in this paper.

In the existing CAD/CAM systems, the triangular Bézier surface and the spline curve are widely used. The shape parameters also have been brought into the triangular surface in [12-14] and the spline curve [15-17]. The method in this paper also can be extended to construct the basis functions of the triangular surface and the spline curve with shape parameters directly, and more details can be seen in our other papers submitted.

\section{Acknowledgments}

The author is very grateful to the anonymous referees for the inspiring comments and the valuable suggestions which improved the paper considerably. This work has been supported by the National Natural Science Foundation of China (no. Y5090377) and the Natural Science Foundation of Ningbo (nos. 2011A610174 and 2012A610029).

\section{References}

[1] G. Farin, "Algorithms for rational Bézier curves," ComputerAided Design, vol. 15, no. 2, pp. 73-77, 1983.

[2] I. Juhász, "Weight-based shape modification of NURBS curves," Computer Aided Geometric Design, vol. 16, no. 5, pp. 377-383, 1999.

[3] L. Piegl, "Modifying the shape of rational B-splines. Part 1: curves," Computer-Aided Design, vol. 21, no. 8, pp. 509-518, 1989.

[4] Q. Chen and G. Wang, "A class of Bézier-like curves," Computer Aided Geometric Design, vol. 20, no. 1, pp. 29-39, 2003.

[5] J. Zhang, "C-curves: an extension of cubic curves," Computer Aided Geometric Design, vol. 13, no. 3, pp. 199-217, 1996.

[6] J. Zhang, F. L. Krause, and H. Zhang, "Unifying C-curves and H-curves by extending the calculation to complex numbers," Computer Aided Geometric Design, vol. 22, no. 9, pp. 865-883, 2005.

[7] X. Wu, "Bézier curve with shape parameter," Journal of Image and Graphics, vol. 11, pp. 369-374, 2006.

[8] X. A. Han, Y. Ma, and X. Huang, "A novel generalization of Bézier curve and surface," Journal of Computational and Applied Mathematics, vol. 217, no. 1, pp. 180-193, 2008.

[9] L. Yang and X. M. Zeng, "Bézier curves and surfaces with shape parameters," International Journal of Computer Mathematics, vol. 86, no. 7, pp. 1253-1263, 2009.

[10] T. Xiang, Z. Liu, W. Wang, and P. Jiang, "A novel extension of Bézier curves and surfaces of the same degree," Journal of Information \& Computational Science, vol. 7, pp. 2080-2089, 2010.

[11] J. Chen and G.-j. Wang, "A new type of the generalized Bézier curves," Applied Mathematics, vol. 26, no. 1, pp. 47-56, 2011.

[12] L. Yan and J. Liang, "An extension of the Bézier model," Applied Mathematics and Computation, vol. 218, no. 6, pp. 2863-2879, 2011.

[13] J. Cao and G. Wang, "An extension of Bernstein-Bézier surface over the triangular domain," Progress in Natural Science, vol. 17, no. 3, pp. 352-357, 2007.

[14] Z. Liu, J. Tan, and X. Chen, "Cubic Bézier triangular patch with shape parameters," Journal of Computer Research and Development, vol. 49, pp. 152-157, 2012.

[15] X. Han, "Quadratic trigonometric polynomial curves with a shape parameter," Computer Aided Geometric Design, vol. 19, no. 7, pp. 503-512, 2002.

[16] X. Han, "Cubic trigonometric polynomial curves with a shape parameter," Computer Aided Geometric Design, vol. 21, no. 6, pp. 535-548, 2004.

[17] X. Han, "A class of general quartic spline curves with shape parameters," Computer Aided Geometric Design, vol. 28, no. 3, pp. 151-163, 2011. 


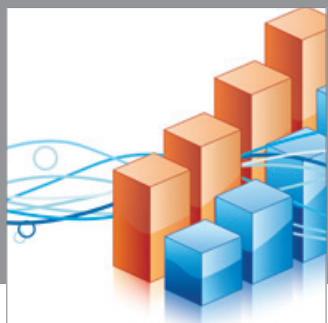

Advances in

Operations Research

mansans

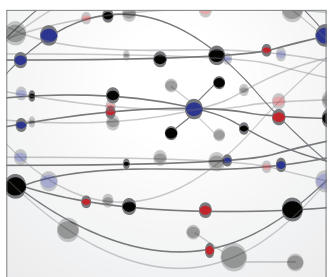

The Scientific World Journal
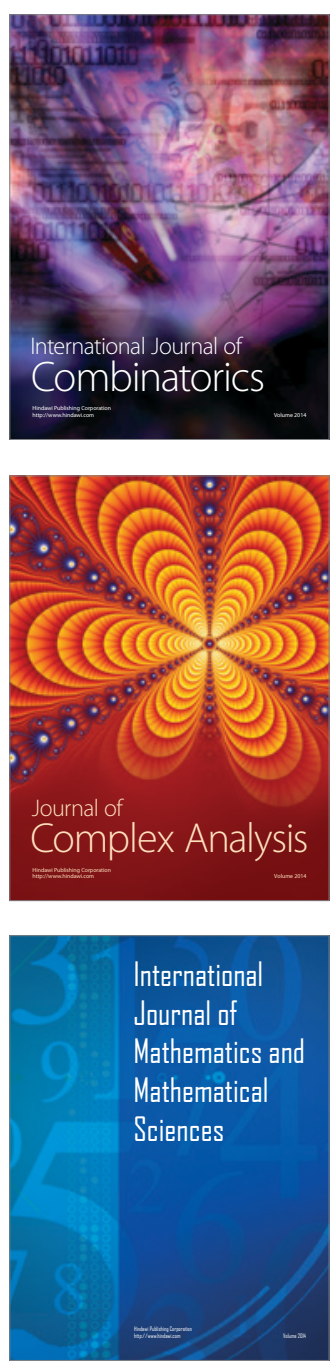
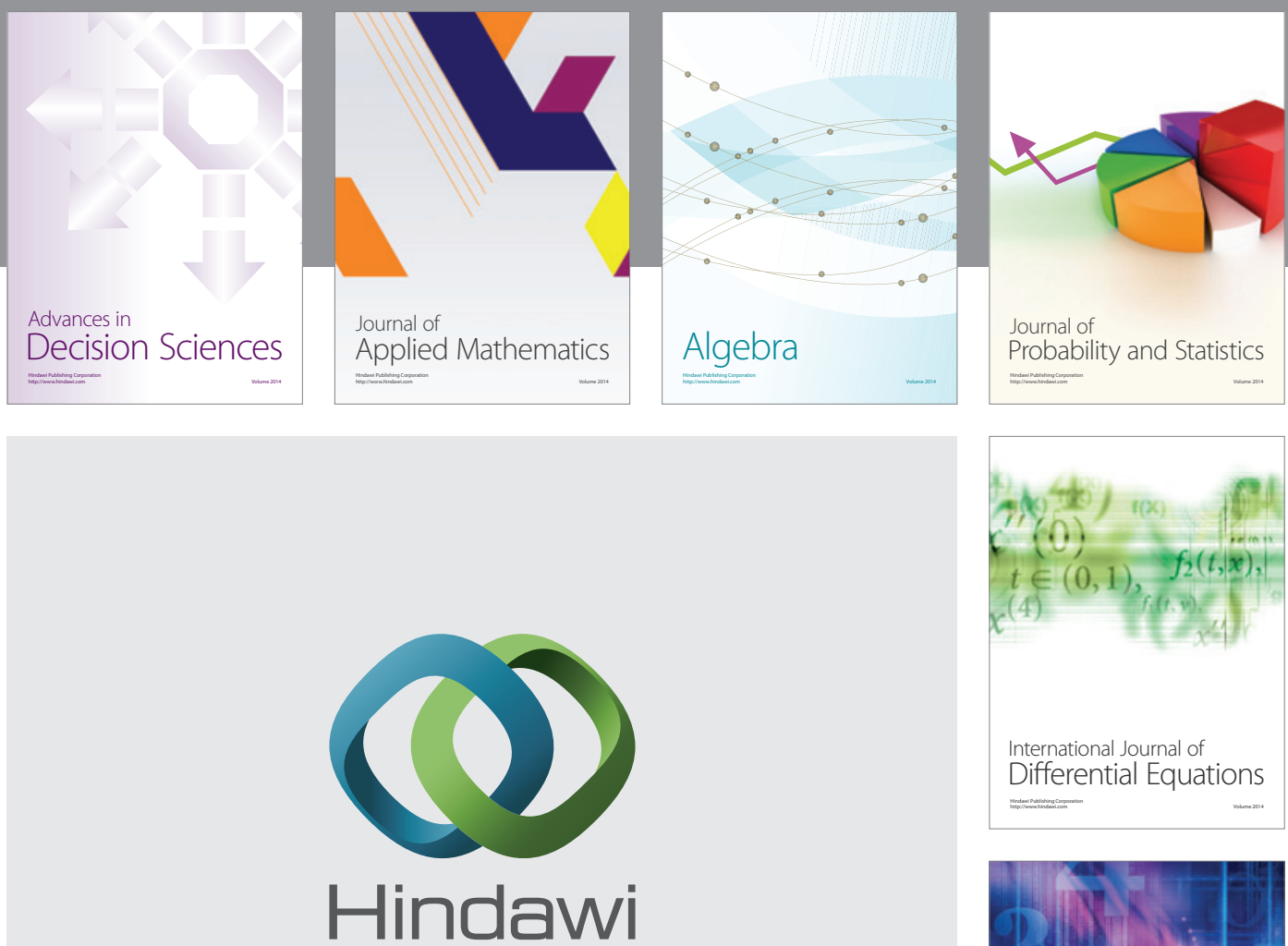

Submit your manuscripts at http://www.hindawi.com
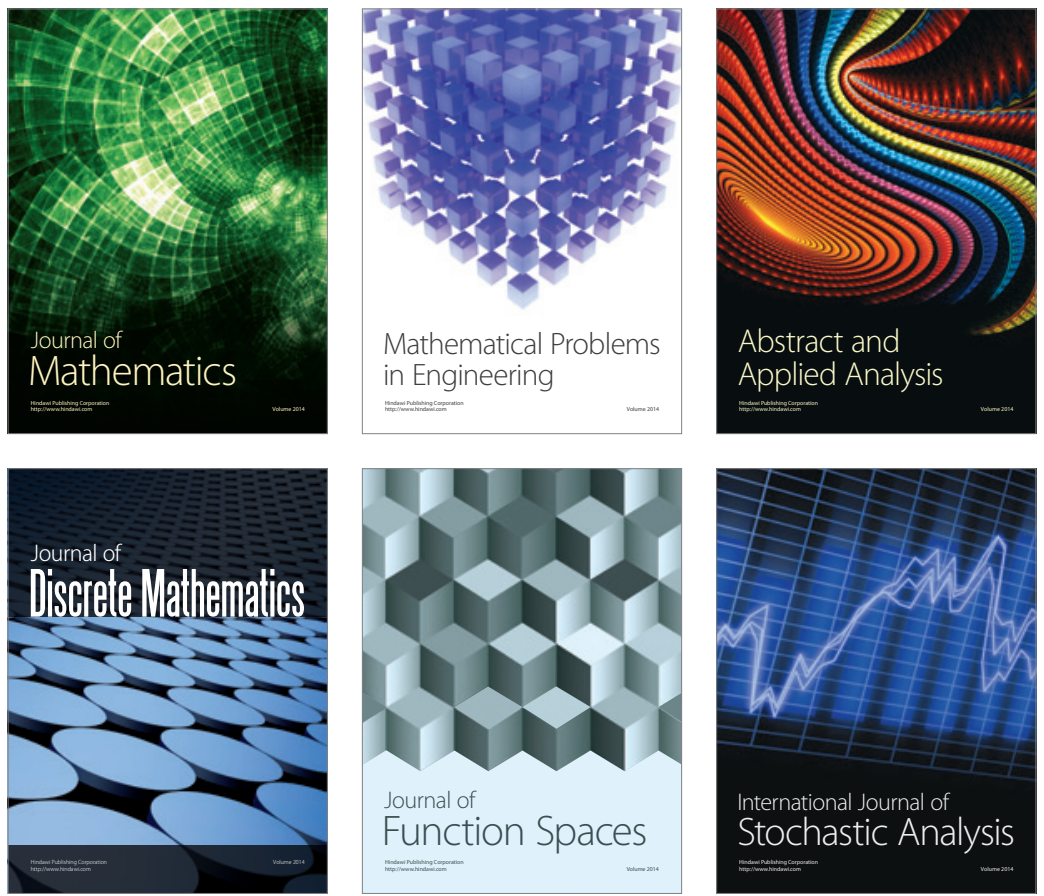

Journal of

Function Spaces

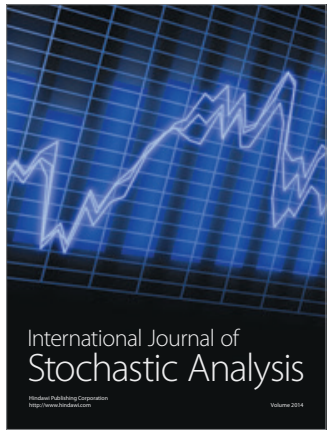

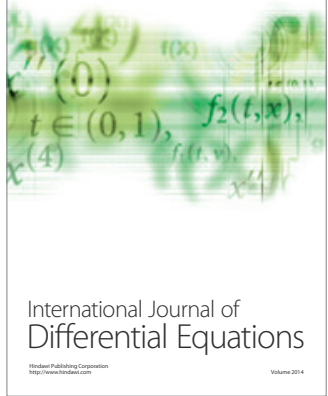
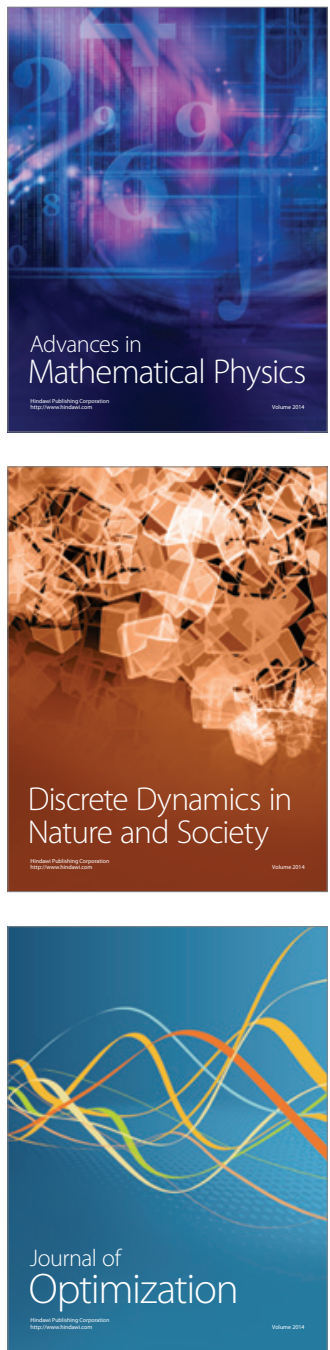